\title{
ANDES

www.scielo.cl

\section{Traqueostomía en niños: experiencia de 10 años en una Unidad de Cuidados Intensivos Pediátricos}

\author{
Pediatric tracheostomy: ten year experience in an Intensive Care Unit \\ Ignacio Oyarzún ${ }^{a}$, María José Conejero ${ }^{\mathrm{b}}$, Rodrigo Adasme ${ }^{\mathrm{c}}$, Carolina Pérez ${ }^{\mathrm{d}}$, Dafne Segalle, \\ Fernando Vulletin ${ }^{\mathrm{f}}$, María Angélica Oyarzúna , Patricio Valle ${ }^{\mathrm{g}}$
}

\begin{abstract}
aDepartamento de Cardiología y Enfermedades Respiratorias Pediátricas, División de Pediatría, Facultad de Medicina, Pontificia Universidad Católica de Chile. Santiago, Chile

bunidad de Pediatría Hospitalaria, Departamento de Pediatría, División de Pediatría, Facultad de Medicina, Pontificia Universidad Católica de Chile. Santiago, Chile

'Escuela de Kinesiología, Facultad de Ciencias de la Rehabilitación, Universidad Andrés Bello. Terapia Respiratoria, Hospital Clínico Red Salud UC Christus. Santiago, Chile

¿División de Pediatría, Facultad de Medicina, Pontificia Universidad Católica de Chile. Santiago, Chile

eDepartamento de Otorrinolaringología, Facultad de Medicina, Universidad de Chile. Hospital San Juan de Dios. Santiago, Chile

‘Sección de Cirugía pediátrica, División de Cirugía, Facultad de Medicina, Pontificia Universidad Católica de Chile. Santiago, Chile

qUnidad de Paciente Crítico Pediátrico, División de Pediatría. Facultad de Medicina, Pontificia Universidad Católica de Chile. Santiago, Chile
\end{abstract}

Recibido: 16 de junio de 2020; Aceptado: 24 de diciembre de 2020

¿Qué se sabe del tema que trata este estudio?

La traqueostomía es un procedimiento habitual en cuidados intensivos pediátricos. La indicación por necesidad de ventilación mecánica (VM) crónica ha aumentado en las últimas décadas.

\section{¿Qué aporta este estudio a lo ya conocido?}

En un centro de derivación en Chile, la indicación de traqueostomía es similar a lo reportado internacionalmente. Niños en VM con menor edad, weaning difícil, genopatía, o con necesidad especiales en salud tienen mayor riesgo de alta con traqueostomía y VM crónica.

\section{Resumen}

La indicación de traqueostomía en pediatría ha cambiado en los últimos 30 años, desde indicaciones agudas y transitorias por obstrucción de la vía aérea a indicaciones programadas y de uso crónico por necesidad de ventilación mecánica (VM) crónica. Objetivo: describir la indicación de traqueostomía y morbilidad asociada al procedimiento, en una cohorte de una década. Pacientes y Método: Estudio descriptivo. Revisión de fichas de egresos hospitalarios (2005-2015) en menores de 15 años traqueostomizados durante su estadía, en una unidad de cuidados intensivos de un hospital universitario. Se evaluaron variables demográficas y clínicas pre y post traqueostomía, estadía en unidad de intensivo, edad al momento de traqueostomía, indicación de ésta, complicaciones precoces ( $<7$ días), complicaciones tardías ( $>7$ días), y mortalidad. Resultados: Se consignaron 59 pacientes traqueostomiza-
Palabras clave:

Traqueostomía; Ventilación Mecánica Crónica; Cuidados Intensivos Pediátricos;

Niños
Correspondencia: Ignacio Oyarzún ijoyarzu@uc.cl

\section{Patricio Valle}

pvalle@med.puc.cl 
dos, 36 (59\%) de ellos menores de 6 meses y 60\% en varones. El 39\% tuvo una genopatía confirmada o en estudio y el $42 \%$ una cardiopatía congénita. Las principales indicaciones fueron compromiso de vía aérea (58\%) y VM crónica (42\%). En el subgrupo de vía aérea, las principales causas fueron estenosis subglótica, parálisis cordal y traqueobroncomalacia, mientras que en el subgrupo de VM crónica las principales indicaciones fueron displasia broncopulmonar y daño pulmonar crónico. No encontramos mortalidad asociada a traqueostomía. El 89\% fue egresado con traqueostomía y el 59\% con VM crónica. Los pacientes de menor edad presentaron mayor probabilidad de alta con traqueostomía y aquellos con mayor número de fallas de extubación previo a traqueostomía, presentaron mayor tasa de egreso con VM. Conclusiones: La traqueostomía es un procedimiento seguro en niños, siendo las causas preponderantes la patología de vía aérea y necesidad de VM crónica. La mayoría de los niños traqueostomizados egresó con traqueostomía y VM crónica. Los niños de menor edad, weaning difícil, genopatía confirmada o sospecha, o con necesidad especiales en salud tienen mayor riesgo de necesitar traqueostomía y VM crónica.
Keywords:

Children;

Tracheostomy;

Pediatric Intensive Care

Unit;

Chronic Mechanical

Ventilation

\section{Introducción}

Se ha reportado que entre un 2,2 a un $19 \%$ de niños hospitalizados en unidades de cuidados intensivos pediátricos que requieren ventilación mecánica (VM) podrían necesitar traqueostomía ${ }^{1}$. Este porcentaje es mayor en grupos de riesgo, como aquellos niños con condiciones crónicas respiratorias, cardiológicas y neurológicas, en que la VM suele ser por períodos más prolongados ${ }^{2,3}$.

Existe un cambio en la indicación de traqueostomía en los últimos 30 años en pediatría: desde su indicación de urgencia por compromiso obstructivo de vía aérea superior, a una indicación programada y de uso por tiempo prolongado por malformaciones con- génitas de vía aérea o dependencia de VM crónica. Si bien la VM crónica es uno de los motivos crecientes de traqueostomía, no existe consenso en su definición. Una de las más aceptadas es la propuesta por Sauthier $\mathrm{M}$ et $\mathrm{al}^{4}$, quienes tras una revisión sistemática definen $\mathrm{VM}$ crónica en pediatría como el uso de $\mathrm{VM} \geq 21$ días consecutivos por más de $6 \mathrm{~h}$ al día, ya sea de manera invasiva o no invasiva.

Los avances de las distintas técnicas quirúrgicas, los cuidados intensivos pediátricos y neonatales, entre otras especialidades, han permitido una mayor sobrevida y calidad de vida de pacientes complejos que pueden agruparse bajo el término NANEAS (niños y adolescentes con necesidades especiales de atención en salud $)^{5}$. Pacientes con malformaciones craneofaciales 
o de la vía aérea, displasia broncopulmonar (DBP), cardiopatías congénitas (CC), enfermedades neuromusculares congénitas o adquiridas, entre otras condiciones, pueden requerir de la traqueostomía como una necesidad especial que les permita optimizar su desarrollo y disminuir morbilidad a largo plazo ${ }^{6,7}$.

En nuestro medio, la evidencia sobre el motivo de indicación de traqueostomía en las unidades de cuidados intensivos es limitada. En un estudio realizado por Ríos Deiddan et al. en un centro terciario en Chile, de un total de 56 niños con traqueostomía en el periodo 2001-2008, la principal indicación fue patología de la vía aérea (70\%) y ventilación prolongada $(30 \%)^{8}$. El objetivo de este estudio es describir las indicaciones de traqueostomía en una unidad de paciente crítico pediátrico (UPC-Ped) de alta complejidad y la morbilidad asociada. Como objetivo secundario, describir las características clínicas pre y post traqueostomía para explorar que factores podrían ser determinantes para el mejor momento de su indicación.

\section{Pacientes y Método}

Estudio descriptivo, de serie de casos, retrospectivo. Se accedió a través de registro computacional a los egresos hospitalarios entre los años 2005 a 2015 de todos los menores de 15 años con diagnóstico de traqueostomía del Hospital Clínico de la Pontificia Universidad Católica de Chile. Se incluyeron en el análisis aquellos pacientes a los que se les realizó la traqueostomía durante la hospitalización. El Hospital Clínico de la Universidad Católica es un centro terciario localizado en un área metropolitana en Santiago de Chile, que además es centro de derivación nacional de cirugía de cardiopatías congénitas.

Los registros clínicos fueron analizados en forma individual, obteniéndose variables demográficas y caracterización clínica previo y posterior a la realización de traqueostomía, estadía en unidad de intensivo, edad al momento de traqueostomía, indicación de ésta. Complicaciones precoces (dentro de 7 días de la realización de traqueostomía), complicaciones tardías (mayor a 7 días de la realización de traqueostomía) y mortalidad.

Análisis estadístico: Se utilizó programa estadístico Stata 15.1 SE (StataCorp, Texas, USA). Se realizó estadística descriptiva según naturaleza de la variable, y prueba de normalidad de Shapiro Wilks para variables continuas. La exploración de asociación entre variables se evaluó con modelos de regresión univariados según naturaleza de variable resultado. Usamos regresión logística para variables dicotómicas; regresión Ologit para ordinales, ambas con reporte de "odds ra- tio" (OR); regresión binominal negativa para variables discretas, con reporte de "incidence risk ratio" (IRR), y regresión lineal simple para continua, con reporte de coeficiente $\beta$. Todos los coeficientes reportados son acompañados por intervalo de confianza al 95\% (IC95\%) y p-value. Para la comparación de diferencia con significación estadística entre variables nominales se utilizó la prueba de Chi cuadrado $\left(\chi^{2}\right)$. Se consideró significación estadística un valor $\mathrm{p}<0,05$ o el cruce del valor neutro por parte del IC95\%. Para el análisis exploratorio de tiempos de hospitalización y variables relacionadas se usó estimación de Kaplan-Meier con comparación entre curvas por prueba de Log-rank y evaluación de la asociación mediante modelo de riesgos proporcionales de Cox reportando Hazard ratio (HR), IC95\% y valor p.

Este estudio fue aprobado por el Comité de Ética de Investigación Clínica de la Pontificia Universidad Católica de Chile (proyecto 16-200).

\section{Resultados}

De un total de 65 egresos hospitalarios con traqueostomía, se analizaron 59 pacientes a los que se realizó traqueostomía durante su hospitalización en este centro. Seis pacientes fueron excluidos ya que tenían traqueostomía al ingreso. La indicación de traqueostomía por grupo y por causa específica se resumen en la figura 1 y tabla 1 respectivamente.

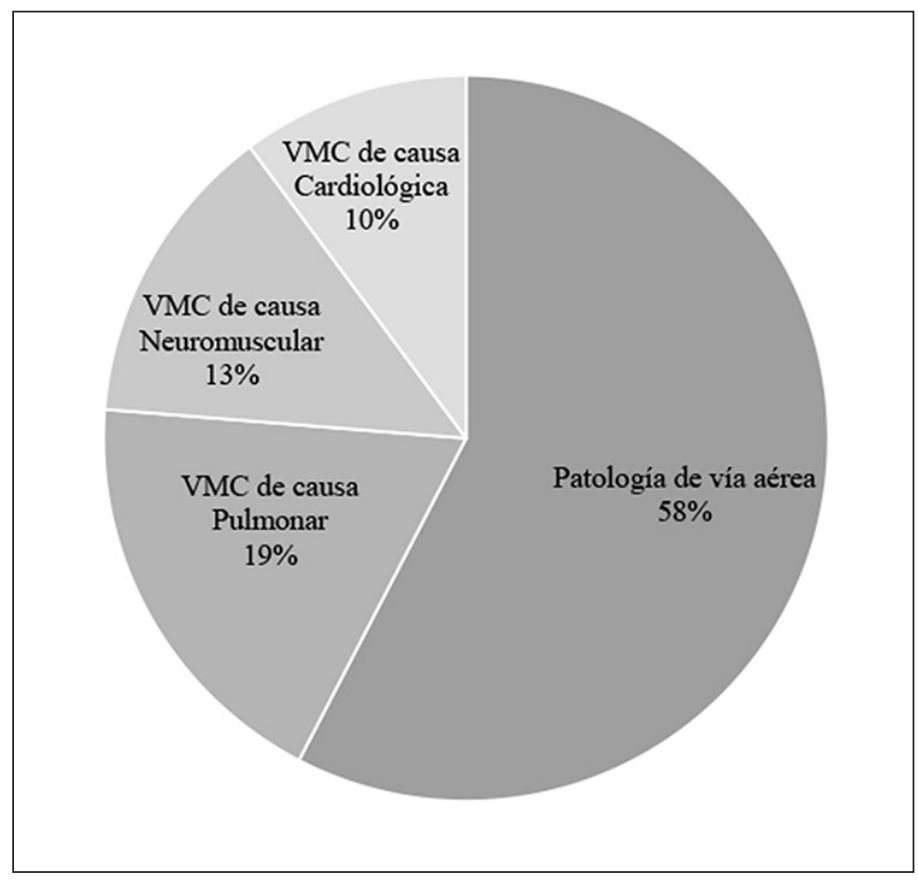

Figura 1. Indicaciones de traqueostomía en pacientes pediátricos. VMC: Ventilación mecánica crónica. 


\begin{tabular}{|c|c|}
\hline Total de pacientes, n (\%) & $59(100)$ \\
\hline Compromiso de vía aérea, $n$ (\%) & $34(58)$ \\
\hline Estenosis subglótica & 8 \\
\hline Parálisis cordal & 8 \\
\hline Traqueobroncomalacia & 8 \\
\hline Hemangioma subglótico & 1 \\
\hline Laringitis post extubación severa & 1 \\
\hline Quiste subglótico & 1 \\
\hline Macroglosia & 1 \\
\hline Atresia Coanas & 1 \\
\hline Estenosis traqueal & 1 \\
\hline Tumor de piso de la boca & 1 \\
\hline Microretrognatia severa & 1 \\
\hline Síndrome linfoproliferativo & 1 \\
\hline Neurofibroma cervical & 1 \\
\hline Ventilación crónica, n (\%) & $25(42)$ \\
\hline Pulmonar, n (\%) & $11(19)$ \\
\hline DBP & 6 \\
\hline DPC & 4 \\
\hline Hipoplasia pulmonar (HDC) & 1 \\
\hline Neuromuscular, n (\%) & $8(13)$ \\
\hline Parálisis diafragmática & 2 \\
\hline Distrofia miotónica & 2 \\
\hline Miopatía centronuclear & 1 \\
\hline Enfermedad de Menkes & 1 \\
\hline Parálisis cerebral & 1 \\
\hline Miopatía paciente critico & 1 \\
\hline Insuficiencia cardiaca, n (\%) & $6(10)$ \\
\hline Hipoplasia de ventrículo izquierdo & 3 \\
\hline Tronco arterioso & 1 \\
\hline DTGA & 1 \\
\hline Atresia tricuspidea & 1 \\
\hline
\end{tabular}

DBP: Displasia broncopulmonar; DPC: Daño pulmonar crónico; HDC: Hernia diafragmática congénita; DTGA: Dextroposición de grandes arterias.
Durante el periodo 2005-2015 el número de nuevas traqueostomías se ha mantenido estable en nuestro centro, siendo entre 6 a 10 intervenciones por año. Durante el periodo 2005-2009 la indicación de traqueostomía por patología de vía aérea corresponde al $65 \%$ y el grupo VM crónica 35\%, en el periodo 2010-2015 corresponden al $54 \%$ y al $46 \%$ respectivamente. La comparación de ambos grupos de indicación de traqueostomía no arrojo diferencias significativas entre los periodos 2005 a 2009 comparado con 2010 a 2015 $(\mathrm{p}=0,707)$.

En la figura 2 se muestran el número de traqueostomías realizadas por grupo etario. Un 59\% corresponde a menores de 6 meses. Un 39\% presentaban el diagnóstico de síndrome genético confirmado o en estudio. Un $42 \%$ presenta cardiopatía congénita, de éstas la cardiopatía congénita compleja más reportada fue hipoplasia de ventrículo izquierdo (HVI). La mediana (rango) de días en VM previo a traqueostomía fue de 28 (1-180) días. Un 52\% de los niños presentó al menos un episodio de falla de extubación. En la tabla 2 se resumen las características clínicas previo a realización de traqueostomía.

Las características clínicas, estadía en UPC-Ped, complicaciones y necesidad de VM y/o traqueostomía al alta se resumen en la tabla 3. Del total de pacientes, 53 tuvieron alta con traqueostomía: 23 con hospitalización domiciliaria y 24 retornaron a su hospital de derivación. Seis pacientes fueron decanulados y dados de alta a su domicilio.

No encontramos mortalidad asociada a la técnica de traqueostomía. Durante su estadía fallecieron seis pacientes. Los fallecimientos ocurrieron a más de 28 días de realizada la traqueostomía y secundario a complicaciones derivadas de sus enfermedades de base. De este grupo, cinco pacientes fueron traqueostomizados

Figura 2. Rangos etarios al momento de realizar traqueostomía en pacientes pe-

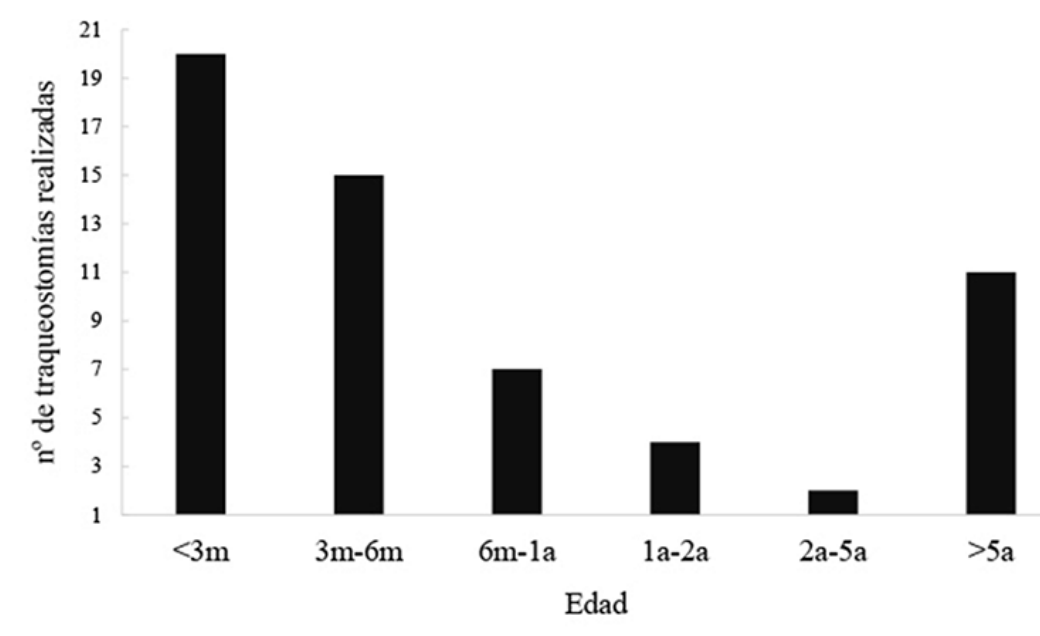


Tabla 2. Caracterización clínica y demográfica previo a realización de traqueostomía

\begin{tabular}{ll}
\hline Total de pacientes, n & 59 \\
Edad de traqueostomía (meses), mediana (min-máx) & $4(0-180)$ \\
Hombres, n (\%) & $39(66)$ \\
Prematurez, n (\%) & $20(34)$ \\
Síndrome genético, n (\%) & $23(39)$ \\
Cardiopatía congénita asociada, n (\%) & $25(42)$ \\
HVI & 6 \\
DAP & 6 \\
Coartación de la aorta & 3 \\
Tetralogía de Fallot & 2 \\
Tronco arterioso & 2 \\
CIA & 2 \\
HVD & 2 \\
CIV & 1 \\
Miocardiopatía hipertrófica & 1 \\
VM, n (\%) & $45(76)$ \\
Causa de VM & \\
Vía aérea & 12 \\
Cardiopulmonar & 23 \\
Neuro-muscular & 10 \\
Días de VM, mediana (min-máx) & $28(1-180)$ \\
Neumonía asociada a VM, n (\%) & $13(29)$ \\
Falla extubación, n (\%) & $45(76)$ \\
No de fallas extubación, mediana (min-máx) & $1(1-5)$ \\
Revisión de VA previa traqueostomía, n (\%) & $43(73)$ \\
\hline
\end{tabular}

HVI: hipoplasia de ventrículo izquierdo; DAP: ductus arterioso persistente; CIA: comunicación interauricular; HVD: hipoplasia ventrículo derecho; CIV: comunicación interventricular; VM: ventilación mecánica; VA: vía aérea.
Tabla 3. Evolución de pacientes pediátricos posterior a la realización de traqueostomía

\begin{tabular}{lc} 
Complicación precoz (< 7 días), n (\%) & $13(22)$ \\
Traqueítis & 11 \\
Granuloma periostomal & 1 \\
Enfisema subcutáneo & 1 \\
Complicación tardía (> 7 días), n (\%) & $18(30)$ \\
$\quad$ Granuloma periostomal & 8 \\
Traqueítis & 9 \\
Decanulación accidental & 1 \\
Mortalidad al día 28, n & 0 \\
Estadía en UPC-Ped (días), promedio ( \pm DE) & $60( \pm 58)$ \\
Traqueostomía al alta, n (\%) & $53(89)$ \\
VM al alta, $n$ (\%) & $35(59)$ \\
\hline UPC-Ped: unidad de paciente crítico pediátrico; VM: ventilación \\
mecánica.
\end{tabular}

por necesidad de VM crónica de causa cardiopulmonar y un paciente por compromiso de vía aérea.

En la exploración de asociaciones reportamos que a mayor edad existe menor probabilidad de alta con traqueostomía, OR 0,99 (IC95\% 0,97-0,99; p < 0,005). En la figura 3 se muestran los distintos grupos de indicación de traqueostomía y su edad de realización, no encontrándose diferencia significativa entre ellos. El tiempo en VM posterior a la realización de traqueostomía es mayor en niños con genopatía confirmada o sospecha, HR 0,56 (95\% IC, 0,36-0,89; $\mathrm{p}<0,005$ ); en el grupo NANEAS, HR 0,55 (95\% IC, 0,32$0,95 ; \mathrm{p}<0,005)$ y en aquellos con mayor número de días de

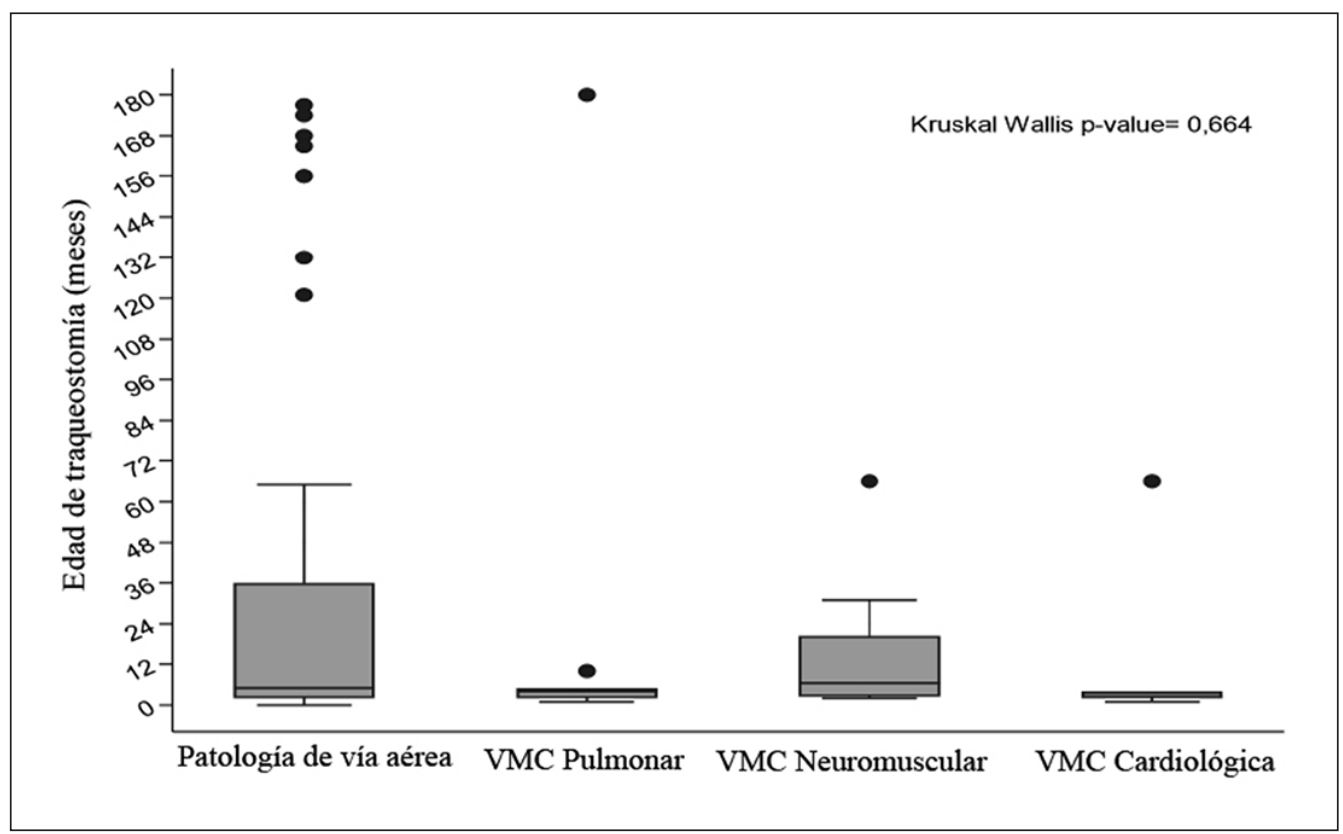

Figura 3. Edad de realización de la traqueostomía según la indicación. VMC: Ventilación Mecánica Crónica. 
ventilación previo a traqueostomía, HR 0,98 (95\% IC, $0,98-0,99), \mathrm{p}<0,005)$. Por otro lado, la probabilidad de alta con VM crónica es mayor en aquellos niños con mayor tiempo en $\mathrm{VM}$ posterior a traqueostomía, OR $1,01(95 \%$ IC, $1,00-1,03 ; \mathrm{p}=<0,005)$ y aquellos niños con mayor número de fallas de extubación previo a la realización de la traqueostomía, OR 5,30 (95\% IC, $1,71-16,5 ; \mathrm{p}=<0,005)$.

\section{Discusión}

En esta serie de pacientes traqueostomizados durante el periodo 2005 a 2015 observamos que, al igual que lo reportado en publicaciones internacionales, la edad de realización de traqueostomía se concentra en el grupo de menores de 6 meses y la indicación por necesidad de VM crece en importancia ${ }^{9-18}$. Gergin et al. describen que entre los años 1984 a 2014 ha existido un cambio en la indicación de traqueostomía en pediatría, desde su indicación principalmente por compromiso de vía aérea y malformaciones craneofaciales, a la indicación por necesidad de VM crónica de causa cardiopulmonar o enfermedad neuromuscular $^{6}$. En nuestro estudio encontramos una tendencia similar. Por otro lado, si tomamos como punto de partida el estudio de Ríos Deidan C et al., realizado en un centro de derivación terciario en Chile entre los años 2001 a 2007, vemos que un $71 \%$ de los niños fueron traqueostomizados por compromiso de vía aérea y un $28 \%$ por necesidad de VM crónica. En el periodo analizado en nuestro estudio, ambos grupos de indicación tienden a ser similares, reafirmándose lo antes señalado.

Las indicaciones específicas de traqueostomía por compromiso de vía aérea encontrada en los pacientes analizados son similares a lo reportado en la literatura. El alto número de parálisis cordales y estenosis subglótica como causa principal de traqueostomía ha sido descrito en centros de derivación de cirugía de cardiopatías congénitas. En estudios realizados en hospitales pediátricos en Estados Unidos por Gorantla et al. ${ }^{19}$ y Khariwala SS et al. ${ }^{20}$, se describe que en el periodo postoperatorio de cardiopatía congénita, un 5\% de los niños operados presenta disfunción cordal, $6 \%$ parálisis cordal bilateral y un 33\% estenosis subglótica. En ambos estudios estas condiciones se relacionaron directamente con la necesidad de traqueostomía.

En los paciente analizados, la principal causa de VM crónica que origino la necesidad de traqueostomía fue el compromiso cardiopulmonar, siendo la DBP o el DPC derivado del periodo neonatal la principal indicación. Se ha descrito hasta un $6,9 \%$ de necesidad de traqueostomía en recién nacidos prematuros menores de 1000 gramos, siendo la DBP su principal indicación, con una media de 112 días en VM previo a la realización de traqueostomía ${ }^{21}$. En aquellos casos en que la insuficiencia cardiaca fue la causa principal de necesidad de VM, encontramos que la hipoplasia de ventrículo izquierdo fue la cardiopatía congénita más frecuente. Hasta un $2,7 \%$ de los niños post operados de cardiopatía congénita tienen dificultad en la suspensión de VM pudiendo necesitar traqueostomía, siendo la hipoplasia de ventrículo izquierdo una de las cardiopatías más relacionadas. La necesidad de traqueostomía en este grupo de pacientes suele acompañarse de anomalías genéticas, anomalías de tráquea y esófago, laringomalacia, alteraciones del diafragma y consecuencias de la cirugía como parálisis cordal o tiempo prolongado de circulación extracorpórea ${ }^{22,23}$.

Una controversia en cuidados intensivos pediátricos y neonatales es definir cuándo se debe realizar una traqueostomía en niños que no logran suspender la VM. En adultos, se ha descrito en distintas revisiones sistemáticas, que la realización de traqueostomía precoz (menor de 7 a 14 días de VM) podría tener beneficios en reducir los días de estadía en UCI, neumonía asociada a VM y mortalidad ${ }^{24,25}$. En Pediatría, una revisión sistemática realizada por AMM Abdelaal et al. ${ }^{26}$ concluye, con un bajo nivel de evidencia, que su realización precoz (menor a 14 días de VM) reduciría la permanencia en VM, estadía en UCI y días de hospitalización, con una tendencia a menor mortalidad. En nuestra serie, la media de días en VM previo a traqueostomía fue alta (28 días). En un estudio multicéntrico liderado por Wakeham MK et al. ${ }^{27}$, de un total de 11.466 niños ingresados a UCI, reportaron una mediana de 14 días en VM previo a traqueostomía con un rango de 5 a 25 días. En este estudio, los predictores independientes de realización de traqueostomía fueron edad menor a 12 meses, 2 o más re-intubaciones, enfermedad cardiopulmonar y mayores días de VM, lo que concuerda con nuestros hallazgos.

Similar a lo reportado por McCrory MC et al. ${ }^{1}$ en su serie de niños traqueostomizados, encontramos que la probabilidad de alta con traqueostomía es mayor en niños de menor edad, y la probabilidad de alta con VM es mayor en aquellos pacientes que presentaron mayor número de fallas de extubación, días en VM previo a la traqueostomía, menor edad, genopatía confirmada o en estudio y categorizados como NANEAS. Debido a que aún la evidencia no es categórica en definir el mejor momento de traqueostomía ${ }^{26}$, creemos que es de utilidad considerar estos predictores de necesidad de traqueostomía y VM al alta al momento de tomar esta decisión.

No encontramos mortalidad relacionada a complicaciones directas de la traqueostomía. En la literatura se reporta una mortalidad relacionada a la técnica entre un 0,7 a 3,6\% ${ }^{28-32}$. JL Funamura et al. ${ }^{31}$ durante un 
seguimiento de 517 niños traqueostomizados entre los años 1984 a 2015, encontraron una mortalidad global de un $16 \%$ en niños traqueostomizados. De este grupo, $5,9 \%$ de los casos falleció durante la misma hospitalización en que se realizó la traqueostomía. Un 0,6\% de mortalidad se relacionó a una complicación de cuidados de traqueostomía como obstrucción de cánula o desplazamiento. Los autores señalan que son las comorbilidades las que influyen en la mortalidad, siendo el grupo de indicación de traqueostomía por VM de causa cardiopulmonar (DBP y CC) el de mayor riesgo de mortalidad, lo que coincide con los casos de fallecimiento encontrados en nuestro estudio.

Un 22\% de los pacientes presentó alguna complicación precoz, siendo traqueítis la principal, seguido de un caso de granuloma y un caso de enfisema subcutáneo. Un 30\% presentó complicación tardía, principalmente traqueítis, granulomas y decanulación accidental. En la literatura se describe una tasa de 18 a 56\% de complicaciones durante la estadía hospitalaria, principalmente neumotórax, neumomediastino, granulomas, infecciones y decanulación accidental ${ }^{11,32}$. Encontramos solo un caso de decanulación accidental, lo cual podría deberse a un subreporte.

Nuestra tasa de decanulación durante la hospitalización fue de un 10\%. La tasa de decanulación durante la misma hospitalización de realización de la traqueostomía es variable en las distintas series publicadas. Se describe entre un 35-75\% según la indicación, siendo precoz en casos de trauma y más tardía en el grupo VM crónica $^{33}$. En nuestro estudio las principales causas de traqueostomía fueron estenosis subglótica y necesidad de ventilación crónica. El primer grupo podría requerir intervenciones quirúrgicas antes de intentar una decanulación, y en aquellos pacientes que requieren VM, se requiere tiempo de rehabilitación y crecimiento para suspender la VM y plantear la decanulación.

Una de las principales fortalezas de este estudio es mostrar la experiencia de 10 años en la realización de traqueostomías en un centro terciario con derivación de pacientes cardioquirúrgicos en Chile. Pudimos objetivar que la necesidad de traqueostomía por compromiso de vía aérea es similar a la necesidad de VM prolongada. Por otro lado, hemos caracterizado un grupo de niños que tendría mayor riesgo de egresar con necesidad de traqueostomía y VM, lo cual nos permite generar hipótesis sobre quienes se beneficiarían de un momento más oportuno para la realización de traqueostomía.

Nuestro estudio tiene limitaciones. Principalmente al ser de carácter retrospectivo puede existir un subreporte de complicaciones relacionadas a traqueostomía. Por otro lado, nuestro seguimiento es hasta el alta de los pacientes o traslado a sus centros de derivación lo que limita evaluar variables de seguimiento pronóstico a largo plazo.

\section{Conclusiones}

Comparado con reportes nacionales previos, la indicación de traqueostomía por necesidad de ventilación crónica ha aumentado, lo cual es similar a lo descrito en series internacionales. La necesidad de traqueostomía por compromiso de vía aérea se mantiene como la principal indicación de traqueostomía en niños, siendo importante además la indicación por uso crónico de VM. Los niños de menor edad, con mayor número de fallas de extubación, genopatía confirmada o sospecha, o con necesidad especiales en salud tienen mayor riesgo de necesitar traqueostomía y VM crónica. Se deben considerar estos factores para una decisión oportuna de la realización de traqueostomía. Es importante sistematizar el seguimiento de niños traqueostomizados para un mejor reporte de complicaciones y morbilidad asociada.

\section{Responsabilidades Éticas}

Protección de personas y animales: Los autores declaran que los procedimientos seguidos se conformaron a las normas éticas del comité de experimentación humana responsable y de acuerdo con la Asociación Médica Mundial y la Declaración de Helsinki.

Confidencialidad de los datos: Los autores declaran que han seguido los protocolos de su centro de trabajo sobre la publicación de datos de pacientes.

Derecho a la Privacidad y Consentimiento Informado: Este estudio ha sido aprobado por el Comité de Ética de Investigación correspondiente, quien de acuerdo a las características del estudio ha eximido el uso del Consentimiento Informado.

\section{Agradecimientos}

Unidad de Paciente Crítico Pediátrico del Hospital Clínico de la Pontificia Universidad Católica de Chile.

\section{Conflicto de intereses}

Los autores declaran no tener conflicto de intereses. 


\section{Referencias}

1. McCrory MC, Lee KJ, Scanlon MC, Wakeham MK. Predictors of need for mechanical ventilation at discharge after tracheostomy in the PICU. Pediatr Pulmonol. 2016;51(1):53-9.

2. Watters KF. Tracheostomy in infants and children. Respir Care. 2017;62(6):799825.

3. Wood D, McShane P, Davis P. Tracheostomy in children admitted to paediatric intensive care. Arch Dis Child. 2012;97(10):866-9.

4. Sauthier M, Rose L, Jouvet P. Pediatric prolonged mechanical ventilation: Considerations for definitional criteria. Respir Care. 2017;62(1):49-53.

5. Flores Cano JC, Lizama Calvo M, Rodríguez Zamora N, et al. Models of care and classification of "Children with special health care needs-CSHCN"': Recommendations from the CSHCN Committee, Chilean Paediatric Society. Rev Chil Pediatr. 2016;87(3):224-32.

6. Gergin O, Adil EA, Kawai K, Watters K, Moritz E, Rahbar R. Indications of pediatric tracheostomy over the last 30 years: Has anything changed? Int J Pediatr Otorhinolaryngol. 2016;87:144-7.

7. Lawrason A, Kavanagh K. Pediatric tracheotomy: Are the indications changing? Int J Pediatr Otorhinolaryngol. 2013;77(6):922-5.

8. Ríos Deidán C., Valenzuela M., Valdivieso J., Correía Dubos G. S. Traquostomía en niños : 7 años de experiencia Hospital Roberto del Río. Revista Pediatría Electrónica 2008;5(3):12-24.

9. Chen $\mathrm{CH}$, Chang JH, Hsu CH, et al. A 12-year-experience with tracheostomy for neonates and infants in northern Taiwan: Indications, hospital courses, and long-term outcomes. Pediatr Neonatol. 2018;59(2):141-6.

10. McPherson ML, Shekerdemian L, Goldsworthy M, et al. A decade of pediatric tracheostomies: Indications, outcomes, and long-term prognosis. Pediatr Pulmonol. 2017;52(7):946-53.

11. Schweiger C, Manica D, Becker CF, et al. Traqueostomia em crianças: uma experiência de dez anos em um centro terciário do sul do Brasil. Braz J Otorhinolaryngol. 2017;83(6):627-32.

12. Franchi R, Baldovino R, Guerra M, et al. Traqueostomía en el niño críticamente enfermo: experiencia de 25 años de una Unidad de Cuidados Intensivos Pediátricos TT - Tracheotomy in a critically ill patient: 25 years experience in the Pediatric Intensive Care Unit. Arch Pediatr Urug. 2016;87(2):95-8.

13. Douglas CM, Poole-Cowley J, Morrissey S, Kubba H, Clement WA, Wynne D. Paediatric tracheostomy-An 11 year experience at a Scottish paediatric tertiary referral centre. Int J Pediatr Otorhinolaryngol. 2015;79(10):1673-6

14. De Trey L, Niedermann E, Ghelfi D, Gerber A, Gysin C. Pediatric tracheotomy: A 30-year experience. J Pediatr Surg. 2013;48(7):1470-5.

15. Özmen S, Özmen ÖA, Ünal ÖF. Pediatric tracheotomies: A 37-year experience in 282 children. Int J Pediatr Otorhinolaryngol. 2009;73(7):959-61.

16. Zenk J, Fyrmpas G, Zimmermann $\mathrm{T}$, Koch M, Constantinidis J, Iro $\mathrm{H}$. Tracheostomy in young patients: Indications and long-term outcome. Eur Arch Oto-Rhino-Laryngology. 2009;266(5):705-11.

17. Graf JM, Montagnino BA, Hueckel R, McPherson ML. Pediatric tracheostomies: A recent experience from one academic center. Pediatr Crit Care Med. 2008;9(1):96-100.

18. Corbett HJ, Mann KS, Mitra I, Jesudason EC, Losty PD, Clarke RW. Tracheostomy-A 10-year experience from a UK pediatric surgical center. J Pediatr Surg. 2007;42(7):1251-4.

19. Gorantla SC, Chan T, Shen I, Wilkes J, Bratton SL. Current Epidemiology of Vocal Cord Dysfunction After Congenital Heart Surgery in Young Infants. Pediatr Crit Care Med. 2019;20(9):817-25

20. Khariwala S, Lee W, Koltai P. Laryngotracheal Consequences of Pediatric Cardiac Surgery. Arch Otolaryngol Head Neck Surg. 2005;131(4):336-9.

21. Overman AE, Liu M, Kurachek SC, et al. Tracheostomy for Infants Requiring Prolonged Mechanical Ventilation: 10 Years' Experience. Pediatrics. 2013;131(5):e1491-6.

22. Prodhan P, Agarwal A, ElHassan NO, et al. Tracheostomy Among Infants With Hypoplastic Left Heart Syndrome Undergoing Cardiac Operations: A Multicenter Analysis. Ann Thorac Surg. [Internet] 2017;103(4):1308-14.

23. Ortmann LA, Manimtim WM, Lachica
CI. Outcomes of Tracheostomy in Children Requiring Surgery for Congenital Heart Disease. Pediatr Cardiol. 2017;38(2):296-301

24. Siempos II, Ntaidou TK, Filippidis FT, Choi AMK. Effect of Early Versus Late or No Tracheostomy on Mortality and Pneumonia of Critically Ill Patients Receiving Mechanical Ventilation: A Systematic Review and MetaAnalysis. Lancet Respir Med. [Internet] 2015;3(2):150-8.

25. Andriolo BN, Andriolo RB, Saconato $\mathrm{H}$, Atallah ÁN, Valente O. Early versus late tracheostomy for critically ill patients. Cochrane Database Syst Rev. 2015;1(1):CD007271.

26. Alkhatip A, Younis M, Jamshidi N, et al. Timing of Tracheostomy in Pediatric Patients: A Systematic Review and MetaAnalysis. Crit Care Med. 2020;48(2):23340.

27. Wakeham MK, Kuhn EM, Lee KJ, McCrory MC, Scanlon MC. Use of Tracheostomy in the PICU Among Patients Requiring Prolonged Mechanical Ventilation. Intensive Care Med. 2014;40(6):863-70.

28. Berry JG, Graham DA, Graham RJ, et al. Predictors of Clinical Outcomes and Hospital Resource Use of Children After Tracheotomy. Pediatrics. 2009;124(2):563-72.

29. Tsuboi N, Ide K, Nishimura N, Nakagawa S, Morimoto N. Pediatric Tracheostomy: Survival and Long-Term Outcomes. Int J Pediatr Otorhinolaryngol. 2016;89:81-5.

30. Lee JH, Smith PB, Quek MBH, Laughon MM, Clark RH, Hornik CP. Risk Factors and In-Hospital Outcomes Following Tracheostomy in Infants. J Pediatr. 2016;173:39-44.e1.

31. Funamura JL, Yuen S, Kawai K, et al. Characterizing Mortality in Pediatric Tracheostomy Patients. Laryngoscope. 2017;127(7):1701-6.

32. Wilcox LJ, Weber BC, Cunningham TD, Baldassari CM. Tracheostomy Complications in Institutionalized Children with Long-term Tracheostomy and Ventilator Dependence. Otolaryngol Head Neck Surg. 2016;154(4):725-30.

33. Funamura JL, Durbin-Johnson B, Tollefson TT, Harrison J, Senders CW. Pediatric tracheotomy: Indications and decannulation outcomes. Laryngoscope. 2014;124(8):1952-8. 